\title{
TESTING A PASSIVE AUTOCATALYTIC RECOMBINER IN THE SURTSEY FACILITY
}

\author{
THOMAS K. BLANCHAT Sandia National Laboratories \\ Reactor Safety Experiments, P.O. Box 5800, Mail Stop 1139, Albuquerque, NM 87185-1139
}
ASIMIOS MALLIAKOS United States Nuclear Regulatory Commission Washington, DC 20555-0001

Performance tests of a scaled passive autocatalytic recombiner $(P A R)$ were performed $(0$ t/ST I Surtsey test vessel at Sandia National Laboratories. Measured hydrogen depletion rate data were obtained and compared with previous work. Depletion rate is most likely proportional to PAR scale. PAR performance in steamy environments (with and without hydrophobic coating) was investigated. The tests determined that the PAR startup delay times decrease with increasing hydrogen concentrations in steamy environments. Tests with placement of the PAR near a wall (as opposed to a center location) yielded reduced depletion rates. Tests at low oxygen concentrations also showed a reduced recombination rate. The PAR repeatedly ignited hydrogen at about 6 mol\% concentration with a catalyst temperature near $940 \mathrm{~K}$. Velocity data at the PAR exhaust were used to calculate the volumetric flow rate through the PAR as a function of the vessel hydrogen concentration.

\section{INTRODUCTION}

Passive autocatalytic recombiners (PARs) have been under consideration in the United States as a combustible gas control system in operating plants and advanced light water reactor (ALWR) containments for design basis accidents. PARs do not require a source of power; instead, they use a catalyst to recombine hydrogen and oxygen gases into water vapor upon contact with the catalyst. The heat produced from the recombination of hydrogen with oxygen creates buoyancy effects that promote the influx of the surrounding gases into the recombiner. The recombination rate of the PAR system needs to be large enough to keep the concentration of hydrogen below detonability limits.

There are several catalytic recombiner concepts under development worldwide. ${ }^{1,2}$ The PAR design tested at Sandia National Laboratories (SNL) has been developed by the NIS Company, Hanau, Germany. Detailed tests and analyses of the NIS PAR have been performed by NIS in cooperation with the Battelle Institute, Frankfurt, and the Technical University, Munich. ${ }^{3}$ Its development has been sponsored by the German utility, RWE Energie. The NIS/RWE PAR device contains flat rectangular cartridges filled with porous spherical ceramic pellets, which are coated with palladium. Between the cartridges, the PAR device has open flow channels that allow heavier particles or aerosols in the atmosphere to flow through with little plugging of the pellet surface.

SNL, under the sponsorship and direction of the U.S. Nuclear Regulatory Commission (USNRC), has conducted an experimental program at the Surtsey test facility to evaluate the performance of PARs. The objectives of the test program were to (1) define the startup characteristics of PARs, (2) confirm the PAR hydrogen depletion rate curve that was provided to 


\section{DISCLAIMER}

This report was prepared as an account of work sponsored by an agency of the United States Government. Neither the United States Government nor any agency thereof, nor any of their employees, make any warranty, express or implied, or assumes any legal liability or responsibility for the accuracy, completeness, or usefulness of any information, apparatus, product, or process disclosed, or represents that its use would not infringe privately owned rights. Reference herein to any specific commercial product, process, or service by trade name, trademark, manufacturer, or otherwise does not necessarily constitute or imply its endorsement, recommendation, or favoring by the United States Government or any agency thereof. The views and opinions of authors expressed herein do not necessarily state or reflect those of the United States Government or any agency thereof. 


\section{DISCLAIMER}

Portions of this document may be illegible in electronic image products. Images are produced from the best available original document. 
the USNRC ${ }^{3}$, (3) define the PAR performance in the presence of steam, (4) evaluate the effect of scale at both low and high hydrogen concentrations, (5) define the PAR performance with and without a hydrophobic coating, (6) determine if the PAR could ignite hydrogen mixtures, (7) define the PAR performance in well-mixed conditions, (8) define the PAR performance in low oxygen environments, and (9) measure the PAR flow rate as a function of hydrogen concentration. This paper describes the configuration of the PAR, the test facility, the instrumentation, the control and data acquisition system, the test conditions, and the test results and analyses.

\section{DESCRIPTION OF EXPERIMENT}

\section{II.A. PAR}

Figure 1 shows the $1 / 4$ scale PAR test module, steel housing, and chimney section. The PAR test module was a scaled version of the prototype PAR that was developed and fabricated by NIS Ingenieurgesellschaft $\mathrm{Mbh} .{ }^{3,4}$ The prototype PAR contained two rows of standard catalytic cartridges ( 44 cartridges per row) and was $1 \mathrm{~m}$ by $1 \mathrm{~m}$. The PAR test module (also manufactured by NIS) contains only one row of standard catalytic cartridges and could be assembled as either a $1 / 2,1 / 4$, or $1 / 8$ scale PAR by removing cartridges and using smaller (length) front and back panels. Note that the $1 / 2$ scale PAR test module configuration has dimensions of $-0.5 \mathrm{~m}$ by $-1.0 \mathrm{~m}$.

Figures 2 and 3 show that the PAR test module housing holds the catalyst cartridges in a vertical direction and guides the air flow. A vertical flow channel with a spacing of about $1 \mathrm{~cm}$ is formed between the cartridges. These flow channels (along with the PAR body or housing) define the flow area for convection of the heat generated by the exothermic reaction of hydrogen recombining with oxygen to produce water vapor. The PAR exit has a chimney with a free cross-sectional area equal to the cross-sectional area through the cartridges. This eliminates downward flow in the PAR and improves the volume flow through the PAR.

The catalyst material is inserted into thin, rectangular cartridges $(0.45 \mathrm{~m}$ length, $0.01 \mathrm{~m}$ wide, $0.20 \mathrm{~m}$ tall). The steel sides of the cartridges are perforated with many slotted-like openings that allow hydrogen to enter into the cartridge. The catalyst is a palladium-coated $(0.5$ weight $\%)$ aluminum oxide pellet with a diameter of about $4-6 \mathrm{~mm}$ and a bulk density of $\sim 0.5 \mathrm{~kg} / \mathrm{L}$. The porous oxide pellet provides a large inside surface area $\left(-100 \mathrm{~m}^{2} / \mathrm{g}\right)$ of palladium. A polymeric hydrophobic coating is placed on each pellet to minimize startup delays due to surface water, either from steam condensation or activation of the containment spray system. NIS states that the hydrophobic coating is probably destroyed when the PAR catalyst exceeds temperatures of about $473 \mathrm{~K}$. The PAR catalyst would reach these temperatures at about 2 mol\% hydrogen gas $\left(\mathrm{H}_{2}\right)$ in cold dry air and about $1 \mathrm{~mol} \% \mathrm{H}_{2}$ in a hot air/steam environment.

\section{II.B. Test Facility}

Figure 4 shows the location of the PAR test module in the Surtsey vessel. The PAR was located at the Surtsey vessel centerline, $\sim 1 \mathrm{~m}$ above the midline elevation (to determine the wall effect, the PAR was moved to within $0.3 \mathrm{~m}$ of the vessel wall for one test). Horizontal and vertical I- 
beams exist in the lower half of the Surtsey vessel but there are no I-beams located directly below the PAR. The horizontal I-beams rest on a rolled steel ring that protrudes about $15 \mathrm{~cm}$ from the wall. The flow area through the beam openings is $47 \%$ of the total Surtsey crosssectional area.

The Surtsey vessel (Fig. 5) is an ASME-approved steel pressure vessel. It has a cylindrical shape with removable, dished heads attached to both ends, and is $3.6 \mathrm{~m}$ in diameter by $10.3 \mathrm{~m}$ high. The Surtsey vessel has a maximum allowable working pressure of $1 \mathrm{MPa}$ at $533 \mathrm{~K}$. A total of twenty $30.5 \mathrm{~cm}$ and $61-\mathrm{cm}$ instrument penetration ports allow steam, noncondensible gas, water, electrical, and video service into and out of the vessel (at six different levels around the perimeter of the vessel). The vessel walls and heads are $3 / 8 \mathrm{in}$. thick and covered with at least 4 in. of fiberglass, or equivalent material, for insulation. The freeboard volume of the Surtsey vessel was $99 \mathrm{~m}^{3}$.

\section{II.C. Instrumentation, Control, and Data Acquisition}

The instrumentation and control equipment and the personal computer-based data acquisition system (DAS) were designed to allow the test conditions to be changed (during the course of a test), based on real-time test results. Real-time plots of the most significant variables provided indications of the PAR heatup and hydrogen depletion during the course of the test. The plots included the vessel hydrogen, oxygen, and steam concentrations; oxygen and hydrogen addition flow rates and total flow; PAR temperatures; vessel gas temperatures from two vertical arrays; vessel pressure; and the velocity of the gas at the PAR inlet and outlet.

Four pressure transducers were used to measure the pressure in the Surtsey vessel. The gas temperature was measured with twenty thermocouples installed in two rakes. The two thermocouple rakes were installed vertically in the vessel, one rake at the vessel centerline (array A) and one rake (array B) located about $0.32 \mathrm{~m}$ from the vessel wall. Ten equally spaced type-K thermocouples ( $1.0 \mathrm{~m}$ spacing) were located on each rake. All type- $\mathrm{K}$ thermocouples were made of $0.254-\mathrm{mm}$ wire with a $1.6-\mathrm{mm}$ sheath. Six type-K thermocouples were installed in the Surtsey vessel steel walls. Five thermocouples measured wall temperature and one thermocouple measured floor temperature. In addition, thermocouples measured the injected oxygen and hydrogen temperatures, both at the respective manifolds and also at each steam/gas diffuser. In order to minimize condensation, steam was mixed with the oxygen and/or hydrogen during each gas injection (described below). The temperature of the inlet steam was also recorded.

A real-time gas mass spectroscopy (GMS) system was used to determine the concentrations of nitrogen, oxygen, and hydrogen in the vessel at four sample points (the PAR inlet, the PAR outlet, near the vessel dome, and near the vessel floor). To ensure representative samples and to minimize the delay time due to purging sample lines, each line was purged for -1 minute prior to sampling. This necessitated a continuous purge of gas out of the vessel. The sample lines and purge rates were sized to allow no more than a $1 \%$ loss (by volume) of gas out of the vessel over the course of a 12-hr test. Since the PAR inlet was the sample point of greatest interest, this point was selected for every other sample (i.e., PAR inlet, PAR outlet, PAR inlet, Surtsey dome, PAR inlet, Surtsey floor, PAR inlet, PAR outlet). Ten to twenty preevacuated $500-\mathrm{cm}^{3}$ gas grab sample bottles were used to collect samples from the vessel. Most of these samples were taken 
at the PAR inlet; however, any of the four gas sample points could have been selected. These gas grab samples were used to independently verify the GMS data post-test.

A high-resolution 1/2-inch charge-coupled device (CCD) color viewed the PAR through a tempered glass window. In addition to the digital camera, an infrared (IR) camera also viewed the PAR to provide visual evidence of deflagrations. Other instrumentation included a hygrometer to measure relative humidity, pitot-tube differential pressure transducers, and a hotwire anemometer to measure the velocity of the gas at the PAR inlet and outlet.

The hydrogen and oxygen gas was supplied to the vessel from separate compressed gas cylinder manifolds. In order to minimize the condensation of vessel steam (in the tests that involved a prototypic air/steam atmosphere), the cold gas entering the vessel was mixed with a small amount of steam in separate diffuser/mixer pipes that were located near the floor of the vessel. For every mole of hydrogen that was added, typically one-half mole of oxygen was also added in those tests at high hydrogen concentrations. Mass flow controllers were used to provide precise metering of the hydrogen and oxygen in the vessel. Two mixing fans were installed in the vessel. They were located on opposite sides of the PAR in openings between the false floor support I-beams; one pointed upward and one pointed downward. The fan placement and speed were designed to minimize perturbation of the PAR and Surtsey vessel flow patterns. The fans were usually operated only when hydrogen was injected and prior to taking gas grab samples (except in the well-mixed tests).

Twelve thermocouples monitored the PAR catalyst temperature at three cartridge locations: PAR middle (and a PAR middle backup), PAR edge, and PAR corner. Three vertical positions for temperature measurement were monitored at each location $(2 \mathrm{~cm}$ from the bottom, the middle, and $2 \mathrm{~cm}$ from the top). These thermocouples were inserted into the cartridges and surrounded by the catalyst pellets. Twelve thermocouples monitored the temperature of the gas in the gap between the cartridges and were located opposite the catalyst thermocouples. Four thermocouples monitored the PAR inlet temperature. Two thermocouples were located at the centerline middle and two at the centerline edge (within $2 \mathrm{~cm}$ of the PAR bottom). Four thermocouples monitored the PAR outlet temperature. Two thermocouples were located at the centerline middle and two were located at the centerline edge (within $2 \mathrm{~cm}$ of the chimney exit). The PAR gas $\Delta T$ (differential temperature) was calculated from the difference of the PAR outlet and inlet average temperatures.

\section{GAS COMPOSITION MEASUREMENTS AND ANALYSES}

The GMS system cannot measure steam concentrations; a dry sample must be presented to the GMS system. In order to achieve this, a condenser and condensate trap (and heated gas inlet lines) were installed on each gas measurement line. This yielded dry-basis gas concentrations; however, to determine wet-basis gas concentrations, the steam fraction must be known. A hygrometer was used to determine the relative humidity $(\mathrm{RH})$. Then the steam concentration $X_{\text {steam }}$ was calculated by,

$$
X_{\text {steam }}=R H \frac{P_{\text {sat }}}{P_{\text {vessel }}} .
$$


where the saturation pressure of steam $\left(P_{\text {sat }}\right)$ was determined from the saturated steam tables using the vessel average gas temperature. The thermocouples on array $B$ were used to determine the vessel average temperature.

The nitrogen-ratio method was used to determine wet-basis gas concentrations as a second independent method. ${ }^{5}$ The nitrogen-ratio method calculates the total number of noncondensible moles. Total moles in the vessel were calculated using ideal gas law relationships. Therefore, the number of steam moles was simply the difference between the total vessel moles and the total noncondensible moles. The steam fraction is the ratio of steam moles to total vessel moles.

\section{TEST MATRIX}

Twenty-two PAR tests were conducted as summarized in Table 1. The PAR was located at the Surtsey vessel centerline in all tests except test PAR-9. The tests using an air environment (no steam) were conducted at an initial pressure of about $0.21 \mathrm{MPa}$. The tests that included steam used a mixture of $0.107 \mathrm{MPa}$ of air and $0.107 \mathrm{MPa}$ of steam, for a total initial pressure of about $0.21 \mathrm{MPa}$. To achieve these conditions, the vessel was sealed with about $0.083 \mathrm{MPa}$ of cold air inside (one Albuquerque atmosphere at about $293 \mathrm{~K}$ ). To achieve a steam atmosphere, the Surtsey vessel was heated internally by injecting saturated steam and removing condensate to obtain a gas temperature of about $375 \mathrm{~K}$.

The first three tests determined the minimum hydrogen concentration at which the PAR begins to recombine, both in cold air (PAR-1 and PAR-2) and in steam atmospheres (PAR-3). These startup tests were all performed at $1 / 2$ PAR scale.

PAR performance at low hydrogen concentrations $(<1-2 \mathrm{~mol} \%)$ was determined in the PAR-4 (at 1/2 PAR scale), the PAR-5 (at 1/4 PAR scale), and the PAR-6 (at 1/8 PAR scale) experiments. The limited hydrogen concentration prevented destruction of the hydrophobic coating.

The effect of the hydrophobic coating was determined in two counterpart tests, PAR-7 and PAR8. Both tests were performed at 1/8 PAR scale and at relatively high hydrogen concentrations. The hydrophobic coating was intentionally destroyed during the PAR-7 experiment. Since a hydrogen burn occurred in PAR-8, a repeat test was necessary to yield performance data at $1 / 8$ PAR scale and high hydrogen concentrations (PAR-8R). The PAR-12 experiment (at 1/4 PAR scale) and the PAR-13 and PAR-13R experiments (at 1/2 PAR scale) yielded high hydrogen concentration performance data for the scaled counterpart.

The PAR-9 experiment was performed at 1/8 PAR scale to determine the effect on performance when the PAR is located near a wall. New cartridges were used and the depletion rate data from PAR-9 were compared with the counterpart test, PAR-6 (also at 1/8 PAR scale but at the vessel center location).

Obtaining hydrogen depletion rates under steady-state, well-mixed conditions was the goal of the PAR-demo1, PAR-demo2, and PAR-demo3 experiments. The mixing fans were operated continuously (at slow speed) throughout most of each test. These experiments were performed with old cartridges that had been last used in the PAR-8R experiment. Steady-state, well-mixed 
depletion rate data were also obtained in the PAR-14 and PAR-15 experiments. New cartridges were installed in the 1/8 PAR scale housing prior to starting the PAR-14 experiment.

The PAR-16 experiment was designed to investigate oxygen limits. The PAR was configured as a 1/8 PAR scale device and was located at the centerline of the Surtsey vessel and about $1 \mathrm{~m}$ above the false floor support I-beams. The Surtsey vessel contained nitrogen at $0.2 \mathrm{MPa}$ at the beginning of the test.

The PP-1 PAR experiment performed in the Surtsey Test Facility was designed with two goals: (1) to perform a counterpart experiment to PAR-9 or PAR-14 and look for similar behavior; i.e., a catalyst temperature spike at about $473 \mathrm{~K}$ believed to be caused by the destruction of the hydrophobic coating, and (2) to obtain PAR flow measurements at various well-mixed hydrogen concentrations. The flow measurement tests were performed in an air/hydrogen atmosphere.

Hydrogen ignition by the PAR was first intentionally tested in the PAR-10 experiment. The ignition of well-mixed hydrogen/air/steam mixtures with both new and used hydrophobic coating on the catalyst pellets was closely examined in the PP-2 PAR experiment. A catalyst temperature greater than $473 \mathrm{~K}$ was reached during the first ignition event (destroying the coating); subsequent ignitions were caused by a catalyst with destroyed coating. The counterpart experiment to PAR-9 or PAR-14 (verifying the catalyst temperature spike at about $473 \mathrm{~K}$, which is suspected to be caused by the destruction of the hydrophobic coating) was achieved in both the PP-1 and PP-2 experiments.

\section{EXPERIMENTAL RESULTS AND ANALYSES}

A general observation from the twenty-two tests is described in the following paragraph. Specific experimental results and detailed test observations are available in NRC ${ }^{6}$ and SNL ${ }^{7,8}$ data reports.

At steady-state operation (without mixing fans), the PAR appeared to generate a convective flow loop in the Surtsey vessel from the PAR outlet to the dome, down the Surtsey wall (until it reached a height near the PAR inlet), and then back to the PAR inlet, as indicated by both the hydrogen concentration and the vessel gas temperature measurements. Since the convection flow pattern did not extend to the Surtsey floor, the vessel was not completely well mixed by the PAR during steady-state operation. The hydrogen taken from the sample point located near the floor typically showed higher concentrations than the other sample points. This indicated that the depletion below the PAR near the floor was lower than that in the upper half of the Surtsey vessel. Also, the convective loop appeared to be driven further downward into the lower half of the Surtsey vessel in tests at higher hydrogen concentrations and/or at a larger PAR scale. 


\section{V.A. Cold Air versus Hot Steamy Startup}

In PAR-1 and PAR-2, recombination started after the first hydrogen addition to about 0.3 mol\% and $0.15 \mathrm{~mol} \%$, respectively. There was essentially no delay in startup. This was shown by increased vessel gas and PAR catalyst temperatures, increased flow at the PAR outlet, and decreasing hydrogen concentrations. In both tests, the PAR was configured for 1/2 PAR scale; the Surtsey vessel was sealed and pressurized with air to about $0.21 \mathrm{MPa}$; and the initial gas temperature was about $295 \mathrm{~K}$. Figure 6 compares hydrogen concentrations throughout the Surtsey vessel with the PAR $\Delta T$. The PAR started recombining after the first hydrogen addition to about $0.15 \mathrm{~mol} \%$, indicated by the increase in PAR $\Delta T$. Note the difference in hydrogen concentration between the vessel floor and the vessel dome at $5.6 \mathrm{hr}$, just before the mixing fans were turned on.

The hydrogen gas injection sequence in the PAR-3 experiment was very similar to the PAR-1 and the PAR-2 experiments. The PAR was configured for 1/2 PAR scale. The Surtsey vessel was sealed and contained cold air at about $0.083 \mathrm{MPa}$. Steam was then added to heat the vessel and the air until a total pressure of about $0.21 \mathrm{MPa}$ was achieved. The gas temperature was about $375 \mathrm{~K}$. The steam concentration at the beginning of the test was about $52 \mathrm{~mol} \%$. The PAR did not give a strong immediate response after the first two hydrogen additions with a 1-hr wait period after each addition $(-0.2 \mathrm{~mol} \%$ each $)$. The PAR started about $2 \mathrm{hr}$ into the test at a concentration of $0.4 \mathrm{~mol} \%$ in PAR-3. However, after a quick injection to $2 \mathrm{~mol} \%$ in the PP-2 air/steam environment, the PAR started in $10 \mathrm{~min}$.

Two general observations regarding PAR startup follow. The first is that the PAR generally started within $10 \mathrm{~min}$ in tests with both cold air atmospheres and with hot air/steam mixtures when hydrogen concentrations were quickly increased to $>1-2 \mathrm{~mol} \%$. In cold air tests, similar fast startups were also seen even with hydrogen additions to only $0.2 \mathrm{~mol} \%$. However, startup delays of 1-2 hr were seen in tests with air/steam atmospheres at low hydrogen concentrations $(0.5-1.0 \mathrm{~mol} \%)$. The delay time decreased when the hydrogen concentrations were increased.

\section{V.B. Startup without the Hydrophobic Coating}

The PAR-7 experiment was designed to operate the 1/8 PAR scale PAR in a steady-state condition by continuously injecting hydrogen to maintain a concentration of about $7 \mathrm{~mol} \%$ (wet basis). The Surtsey vessel was sealed and contained air at about $0.083 \mathrm{MPa}$. Steam was then added to heat the vessel and the air until a pressure of about $0.21 \mathrm{MPa}$ was achieved. The gas temperature was about $375 \mathrm{~K}$. The steam concentration at the beginning of the test was about 54 mol\%. The PAR started about $7 \mathrm{~min}$ after the first hydrogen addition. In PAR-7, the hydrogen concentration reached about $8 \mathrm{~mol} \%$ and the catalyst temperature reached $1000 \mathrm{~K}$, which destroyed the hydrophobic coating.

The PAR-8 experiment was designed to replicate the conditions of PAR-7 and to determine if the lack of the hydrophobic coating would delay the startup in a steam environment. The PAR was configured as a 1/8 PAR scale device (with the same cartridges used in PAR-7) and located in the middle of the Surtsey vessel. The first hydrogen addition replicated the first hydrogen addition in PAR-7. The PAR started about $18 \mathrm{~min}$ after the first hydrogen addition. Based on 
these results, it appeared that the lack of the hydrophobic coating had little effect in delaying the PAR startup at high hydrogen concentrations.

\section{V.C. Hydrogen Depletion Rate Calculation}

Hydrogen depletion rates are used to measure the performance of a PAR. This rate is usually determined as a function of the hydrogen concentration in the vessel. Depletion rate analyses can also be used to show the effect of various factors, such as PAR location, oxygen concentration, and catalyst poison, on PAR performance.

The following methodology was used to determine the depletion rate. First, the time-dependent amount of hydrogen in the Surtsey vessel (in moles) was determined by multiplying the average hydrogen concentration by the total number of moles in the Surtsey vessel. The average hydrogen concentration was assumed to be that measured by the gas mass spectrometer at the PAR inlet sample point, adjusted by the steam fraction (a well-mixed assumption). The total number of moles in the vessel was calculated using the ideal gas law, with an average temperature determined from the array $\mathrm{B}$ thermocouples. The depletion rate was then determined by calculating the reduction in hydrogen, using a smooth curve fit of the hydrogen data from the steady-state depletion interval after the hydrogen additions were stopped. The calculated depletion rate was then plotted against the measured PAR inlet sample point wet-basis hydrogen concentration.

The hydrogen concentrations measured at the four sample locations diverged from some initially equal value over the course of the unmixed tests. This was because the mixing fans were turned off while depletion rate data were taken and the PAR flow was not sufficient to maintain a mixed condition in the vessel. The methodology used to determine the depletion rate assumed that the vessel was well mixed; this introduces some error because the average hydrogen concentration was not actually measured and cannot be calculated since the local steam concentrations were not known. However, there was typically little change in the hydrogen concentration measured at the PAR inlet location after mixing (see Fig. 6 at $5.6 \mathrm{hr}$ ). Note that the depletion rates determined in the unmixed tests could overpredict the hydrogen concentration if the concentration at the PAR inlet sample was lower than the true average value, and underpredict the hydrogen concentration if the inlet concentration was higher than the true average value.

\section{V.D. Hydrogen Depletion and PAR Scale Effect}

PAR performance and the effects of scale were determined at both low and high hydrogen concentrations. All tests started with a vessel pressure of about 2 bar, with approximately 50/50 mixtures of air and steam. Figure 7 shows the PAR performance with low hydrogen concentrations (<0.7 mol\%) at three scales: 1/2 PAR scale (PAR-4), 1/4 PAR scale (PAR-5), and 1/8 PAR scale (PAR-6). Note that the $1 / 2$ scale depletion rate is $\sim 4$ times the $1 / 8$ PAR scale depletion rate. Figure 8 shows the PAR performance with high hydrogen concentrations (1-6 mol\%) and at three scales: 1/2 PAR scale (PAR-13), 1/4 PAR scale (PAR-12), and 1/8 PAR scale (PAR-8R). As with the low hydrogen concentration data, the $1 / 2$ PAR scale depletion rate is $\sim 4$ times the $1 / 8$ PAR scale depletion rate. 
Hydrogen depletion by the NIS prototype PAR can be predicted using the Fischer ${ }^{9}$ correlation or the Sher ${ }^{10}$ depletion rate model. Both methods yield essentially identical results. The Sher model also assumes that depletion rate is directly proportional to scale, which is supported by the PAR data in Figs. 7 and 8. A simple scale factor can be used to normalize the data, based on the ratio of the prototype PAR inlet flow area to the scaled PAR inlet flow area. Neither model has corrections for scaled heat losses.

Fischer found that the full-scale prototype PAR experiment results obtained at Battelle were best fit by assuming that the PAR flow rate is a power law function of the volume fraction of hydrogen,

where

$$
Q=0.67 C_{H}^{0.307}
$$

$Q \quad=\quad$ full-scale prototype PAR steady-state volumetric flow $\left(\mathrm{m}^{3} / \mathrm{s}\right)$

$C_{\mathrm{H}}=$ hydrogen volume fraction in the containment

This equation was based on the prototype full-scale PAR. The hydrogen removal or depletion rate (in $\mathrm{kg} / \mathrm{hr}$ ) can be calculated using

where

$$
R=\varepsilon Q \rho_{H}
$$

$\varepsilon \quad=\quad$ the prototype PAR efficiency factor for hydrogen removal $(0.85)$

$Q=$ volumetric flow rate of containment gas through the PAR $\left(\mathrm{m}^{3} / \mathrm{hr}\right)$

$\rho_{\mathrm{H}}=$ mass density of hydrogen in the PAR $\left(\mathrm{kg} / \mathrm{m}^{3}\right)$

The density of the hydrogen gas at the PAR inlet can be estimated using

where

$$
\rho_{H}=X_{H} \frac{P_{\text {vessel }}}{R_{H} T}
$$

$X_{\mathrm{H}}=$ wet-basis hydrogen molar fraction at the PAR inlet

(estimated using dry-basis measurement and either Eq. 1 or nitrogen-ratio method)

$P_{\text {vessel }}=\quad$ vessel pressure $(\mathrm{Pa})$

$R_{\mathrm{H}} \quad=\quad$ hydrogen gas constant (4124)

$T=$ temperature at the PAR inlet (K)

However, if the PAR consumes hydrogen in only a small portion of the total vessel volume (the "participating" volume), the depletion rate calculation can overpredict consumption if the vessel is not well mixed at all times. The depletion rate measurements may appear to become somewhat scale dependent since tests with larger scale and/or higher concentrations appear to deplete larger pockets of hydrogen within the total vessel volume. Temperature measurements from the vessel gas array thermocouples indicate that the convective loop ends at different heights below the PAR for different test conditions, possibly revealing that larger pockets of hydrogen are depleted at larger scale and at higher hydrogen concentrations. 
Figures 9 and 10 show the scaled depletion rate data normalized to full scale by applying a simple scale factor ( $\mathrm{x} 2$ for 1/2 PAR scale, $\mathrm{x} 4$ for $1 / 4$ PAR scale, and $\mathrm{x} 8$ for 1/8 PAR scale). Figure 9 shows that the depletion rates for tests with low hydrogen concentrations are indeed directly proportional to scale, implying that the participating volume is unaffected by flow through the PAR. However, Fig. 10 shows slightly lower depletion rates for larger scale when the hydrogen concentrations are high.

Figures 9 and 10 also show the Fischer correlation at three conditions, 1 bar air-only, 1 bar steam plus 1 bar air, and 2 bar steam plus 1 bar air. Predictions at 1 bar are only presented to highlight the physics; depletion of hydrogen by the PAR is a mass diffusion process driven by density gradients. The Surtsey low hydrogen concentration data showed greater hydrogen depletion rates than those predicted by the Fischer correlation ( 2 bar test pressure). At high hydrogen concentrations (with the steady-state depletion rate condition typically between 2-3 bar pressure), the 2- and 3-bar Fischer predictions bracket the normalized Surtsey test data. Different PAR designs must have different performance curves. Note that the Fischer correlation was based solely on the NIS prototype PAR design, which did not use the additional $0.5-\mathrm{m}$ tall chimney used in the SNL PAR tests.

\section{V.E. Depletion Rates Under Well-Mixed Conditions}

Figure 11 shows depletion rate comparisons (normalized to full scale) between unmixed and well-mixed conditions. The PAR-8R test was conducted in a hydrogen/air/steam mixture at 2 bar pressure and without mixing fans. The PAR-15 and the PAR PP-1 tests both occurred in well-mixed hydrogen/air mixtures at 2 bar pressure (no steam). In all three tests, the PAR was first operated at a steady-state well-mixed condition by continuously injecting hydrogen. In all three tests the PAR was configured as a 1/8 PAR scale device and was located at the centerline of the Surtsey vessel and about $1 \mathrm{~m}$ above the false floor support I-beams. The mixing fans were operated continuously at a slow speed throughout most of the PAR-15 and PP-1 tests, yielding uniform depletion of the entire Surtsey vessel volume.

The depletion rate calculation assumes that the entire Surtsey volume is being depleted and that the hydrogen concentration in the vessel is uniformly the same concentration as that measured by the GMS at the PAR inlet sample point. The comparison above shows little difference between the unmixed and well-mixed depletion rates with a 1/8 PAR scale with hydrogen concentrations between 1 and 3 mol\%. Mixing effects at a large scale cannot be compared because there are no counterpart data.

\section{V.F. Catalyst Temperature and PAR $\triangle T$ as Functions of Hydrogen Concentration}

The catalyst temperature and the difference in temperature between the PAR inlet and the PAR outlet both provide evidence of PAR performance. Figures 12 and 13 show PAR catalyst temperatures and PAR $\Delta T$ as a function of hydrogen concentration during the steady-state depletion intervals, respectively. The catalyst temperature increased about $96 \mathrm{~K}$ for each $1 \%$ of hydrogen concentration, regardless of the starting temperature. The PAR $\Delta T$ increased about 46 $\mathrm{K}$ for each $1 \%$ of hydrogen concentration. The initial temperature of the vessel gas mixture or the scale of the PAR had little effect on the temperature increases. The PAR $\Delta T$ increase was 
much less than the Electric Power Research Institute (EPRI) PAR results (80 K for each 1 mol\% of hydrogen concentration). Again, note that the SNL PAR tests were performed with an additional $0.5-\mathrm{m}$ tall chimney section. This section probably increased flow through the PAR and reduced the $\Delta T$.

The above PAR $\triangle T$ correlation and the catalyst temperature correlation can be used to estimate the hydrogen concentration in the vessel. Figure 14 shows good agreement between measured and predicted hydrogen concentrations after the PAR started and reached steady-state operations in the PP-1 experiment.

\section{V.G. Wall Effect}

Two counterpart experiments were performed to determine if the placement of a PAR near a wall would affect performance, compared with placement of a PAR in an open volume. Both tests were performed at 1/8 PAR scale and at 2 bar pressure, with a 50/50 mixture of air and steam. Figure 15 shows the depletion rates for PAR-6 (center location) and PAR-9 (wall location). Mixing fans were off in both tests during the depletion rate measurements. The wall clearly appeared to have an effect, yielding a smaller depletion rate than the open-volume test. This was probably because a downflow near the wall impeded natural convective flows at low hydrogen concentrations and/or small PAR scale.

\section{V.H. Oxygen Limit Effect}

The PAR appeared to slow down in the PAR-7 experiment, with hydrogen concentration increasing and oxygen concentration decreasing. In PAR-7, a "restart" occurred when oxygen was added. The PAR-8R test was performed under similar conditions; however, oxygen was maintained near $12 \mathrm{~mol} \%$ throughout the test. Figure 16 shows the depletion rates for PAR-7 (low oxygen) and PAR-8R (excess oxygen). Above $4 \mathrm{~mol} \%$ hydrogen concentrations, the hydrogen depletion rate in PAR-7 was substantially smaller than in PAR-8R. Reduced oxygen levels did not affect the PAR-7 depletion rate data below $3 \mathrm{~mol} \%$ hydrogen concentration because oxygen was added as the hydrogen concentration was about 4 mol\% and decreasing.

PAR performance at limited oxygen concentrations was intentionally tested in PAR-16. The Surtsey vessel was sealed and inerted with nitrogen. At the beginning of the test, the nitrogen and oxygen concentrations were 99.7 and $0.3 \mathrm{~mol} \%$, respectively, as determined by the on-line gas mass spectrometer. The vessel pressure was about $0.22 \mathrm{MPa}$. The gas temperature was about $274 \mathrm{~K}$. The mixing fans were operated continuously (at slow speed) throughout most of the test. After the mixing fans were started, hydrogen was added to about $1.8 \mathrm{~mol} \%$. Slight increases in PAR temperature indicated that the PAR started recombining with oxygen concentration at $0.2 \mathrm{~mol} \%$. Additional increases in temperature occurred with incremental oxygen additions. Catalyst temperature did not increase after a hydrogen addition from 1.4 mol\% to $2.4 \mathrm{~mol} \%$, with the oxygen level at about $0.7 \mathrm{~mol} \%$. The PAR-16 experiment determined that reduced oxygen concentrations resulted in degraded PAR performance. At two points during the experiment the same hydrogen concentration $(3.0 \mathrm{~mol} \%)$ occurred at different oxygen concentrations $(1.5 \mathrm{~mol} \%$ and $4.0 \mathrm{~mol} \%)$. The catalyst temperature was $380 \mathrm{~K}$ at 1.5 
mol\% oxygen and $520 \mathrm{~K}$ at $4.0 \mathrm{~mol} \%$ oxygen. Note that catalyst temperature is a direct measurement of PAR performance.

Figure 16 also shows the hydrogen depletion rate as a function of hydrogen concentration at various oxygen concentrations (A through $G$ ) during the PAR-16 experiment. Note that the PAR depleted hydrogen at very low oxygen concentrations; however, oxygen starvation certainly yielded reduced depletion rates (i.e., low oxygen concentrations limit the amount of hydrogen that can recombine to something less than stoichiometric levels). It is interesting that the depletion rate appeared to decrease slightly when the fans were turned off near the end of the test after the last oxygen addition $(\mathrm{G})$, with the oxygen concentration at $3 \mathrm{~mol} \%$ and decreasing.

\section{V.I. Hydrogen Ignition by the PAR}

Deflagrations of hydrogen were seen in the air/steam tests (PAR-8, PAR-10, PAR-13, and PAR13R experiments) and also in the air-only tests (PAR-demo1, PAR-demo2, and PAR-demo3). There appeared to be two separate ignition modes.

The first ignition mode was probably related to the PAR catalyst hot surface. Surface temperatures greater than $1000 \mathrm{~K}$ are in the range for hot surface ignition in 50 mol\% steam environments. A large deflagration was seen in PAR-8 after hydrogen was increased from $\sim 9$ mol\% to $\sim 11 \mathrm{~mol} \%$. The peak catalyst temperature was about $1100 \mathrm{~K}$. A large deflagration also was seen in PAR-10 immediately after hydrogen was quickly increased to about $-12-13$ mol\% (the measured peak hydrogen concentration was only $\sim 11$ mol\%, probably because the sample system did not have adequate time to purge the sample line and capture the true concentration). The peak catalyst temperature was about $950 \mathrm{~K}$.

Ignition also occurred at much lower hydrogen concentrations (4-5 mol\%) and with peak catalyst temperatures in the range of $600-800 \mathrm{~K}$. These surface temperatures are too low to ignite hydrogen. In PAR-13 and PAR-13R, a combination of new and old catalyst cartridges was used. Videos of these and other tests showed small, whitish-looking particles floating in the vessel whenever new cartridges (with undamaged coating on the catalyst pellets) were heated to temperatures above $500 \mathrm{~K}$. In the PAR-13 video, a burn started in the PAR immediately after a burst of these small whitish-looking particles ejected out of the chimney. It is important to note that the cartridges used in the PAR- 8 and the PAR-10 experiments were previously subjected to high concentrations of hydrogen and that the hydrophobic coating was mostly destroyed before these tests were performed.

In the PAR-demo tests, flame fronts were seen descending from above the PAR, ascending from below the PAR, and also exiting from the PAR chimney. Some of the ignitions may have been caused by extraneous sources, such as the quartz lamps or friction-induced static discharges. However, since ignition still occurred in later tests when these extraneous sources were eliminated, the actual ignition source remained unknown.

The PP-2 experiment was designed to rigorously evaluate the hydrogen ignition characteristics of a PAR in a well-mixed air/steam environment with both new and used hydrophobic coating on the catalyst pellets. The PAR was configured at 1/8 PAR scale using new cartridges containing 
intact hydrophobic coating. The PAR was located at the centerline of the Surtsey vessel and about $1 \mathrm{~m}$ above the false floor support I-beams. Pressure, temperature, and gas sampling instrumentation were as in previous tests, with the exception that the X-Y translator table, hotwire anemometer, lights, and hygrometer were either electrically disconnected or physically removed from the Surtsey vessel to ensure that all extraneous electrical sources were removed. The CCD and the IR cameras recorded the PAR and surrounding views. The Surtsey vessel was sealed and contained air at about $0.084 \mathrm{MPa}$. Steam was added until the vessel pressure was about $0.22 \mathrm{MPa}$ and the temperature was $375 \mathrm{~K}$. This yielded a steam concentration of $50 \mathrm{~mol} \%$ and an air concentration of $50 \mathrm{~mol} \%$. Operation of the mixing fans ensured a well-mixed condition. At the beginning of the test, about $200 \mathrm{~mol}$ of hydrogen were injected to achieve a concentration of $2 \mathrm{~mol} \%$ in the Surtsey vessel. The intent was to let the PAR start and reach the point where the new catalyst typically shows a spike increase in catalyst temperature, thought to be associated with the destruction of catalyst coating.

Following the temperature spike, hydrogen was injected at a rate slightly above the PAR consumption rate to slowly raise the hydrogen concentration in the Surtsey vessel to flammable levels until a deflagration occurred. Following a brief pause to collect data and/or refresh the hydrogen manifold, the ignition cycle was repeated until the hydrogen gas supply (twenty $44 \mathrm{~L}$ gas cylinders) was exhausted. Gas grab samples were taken prior to and immediately after most ignition events to verify the on-line gas mass spectrometer data.

Four hydrogen deflagration events (burns) occurred in the Surtsey vessel under closely repeatable conditions of hydrogen concentration and PAR temperatures. Figure 17 plots the vessel hydrogen concentration, the hydrogen addition history, and the average catalyst temperature for the PP-2 test. The catalyst temperature spike $(0.6 \mathrm{hr})$ followed by the four deflagration events (indicated by sharp decreases in temperature and concentration) are evident. Note that a hydrogen burn did not occur at $t=6.15 \mathrm{hr}$, with a peak catalyst temperature of about $960 \mathrm{~K}$ and $7.8 \mathrm{~mol} \%$ hydrogen. The gas mixture probably became nonflammable due to the steam concentration approaching $63 \mathrm{~mol} \%$. The PAR catalyst was new prior to starting this test. Due to the high, sustained temperature from the first burn, the catalyst coating was considered to be destroyed for the three subsequent burns. Table 2 summarizes (for all tests in which deflagrations occurred) the conditions in the vessel and in the PAR immediately prior to the burns.

A video from the IR camera showed repetitious bursts of hot, glowing particles. The particles ejected from the PAR chimney exited at intervals of a few minutes when hydrogen concentration increased above $3.5 \mathrm{~mol} \%$, until a burn occurred. These particles floated in the vessel and moved with the gas flow streams. After each burn, the particles disappeared from view as they cooled down with the decreased hydrogen concentration. As the hydrogen concentration increased prior to the next burn, the particles became hotter and brighter in the IR camera view. The higher hydrogen concentration promoted new repetitious particle bursts from the PAR chimney. Figure 18 shows a sequence of IR images during the second burn in PP-2. At $0.5 \mathrm{~s}$, a large burst of hot particles ejected from the chimney exit. At $1.0 \mathrm{~s}$, the hydrogen burn clearly initiated in the particle cloud. At $1.5 \mathrm{~s}$, a global deflagration of the vessel hydrogen commenced. The hydrogen deflagration event was easily discernible by the IR camera since the deflagration was much hotter than the temperature of the glowing particles or the PAR housing [the intensity 
of the heat from the burn forced the electronic aperture to automatically stop down (partially close)]. With the vessel internal lighting disconnected, the CCD camera could not see the PAR body or the hydrogen combustion events; however, the burst of particles from the chimney exit and suspension of glowing particles in the Surtsey vessel were visible.

\section{V.J. PAR Flow Measurements}

The main goal of the PP-1 experiment was to determine the flow characteristics of a $1 / 8$ scale $\mathrm{PAR}$ at various hydrogen/air concentrations. The PAR was configured at $1 / 8$ scale using new cartridges containing intact hydrophobic coating. The PAR was located at the centerline of the Surtsey vessel and about $1 \mathrm{~m}$ above the false floor support I-beams. The two pitot tubes and the hot-wire anemometer were mounted on an X-Y translator table that allowed a full traverse of the PAR at the chimney outlet. The Surtsey vessel was sealed and contained air at about $0.22 \mathrm{MPa}$ and about $286 \mathrm{~K}$. First, the PAR was operated at a steady-state, well-mixed condition by slowly injecting hydrogen and oxygen and continuously operating the mixing fans. At about $\mathrm{t}=4.3 \mathrm{hr}$ and at $1.6 \mathrm{~mol} \%$ hydrogen, the catalyst temperature spiked upward and quickly returned to its previous value (about $460 \mathrm{~K}$ ), replicating previous behavior (PAR-9 and PAR-14). The hydrogen concentration was then increased above $3.5 \mathrm{~mol} \%$ to obtain flow data. Figure 19 shows that four sets of velocity measurements (indicated by the instrumentation traverses at the chimney exit) were taken in well-mixed conditions as the PAR recombined the hydrogen gas in the Surtsey vessel:

1) at $\mathrm{t}=5.535 \mathrm{hr}$ and hydrogen concentration at $3.15 \mathrm{~mol} \%$, mean velocity $1.09 \mathrm{~m} / \mathrm{s}$,

2) at $t=6.025 \mathrm{hr}$ and hydrogen concentration at $2.10 \mathrm{~mol} \%$, mean velocity $1.00 \mathrm{~m} / \mathrm{s}$,

3) at $t=6.480 \mathrm{hr}$ and hydrogen concentration at $1.52 \mathrm{~mol} \%$, mean velocity $0.78 \mathrm{~m} / \mathrm{s}$,

4) at $\mathrm{t}=7.510 \mathrm{hr}$ and hydrogen concentration at $0.83 \mathrm{~mol} \%$, mean velocity $0.45 \mathrm{~m} / \mathrm{s}$.

Each traverse consisted of 32 measurements along the PAR chimney centerline, 17 in the $\mathrm{x}$ direction (side-to-side) and 15 in the y direction (front-to-back). In order to determine the mean full-field velocity, it was assumed that the one measured velocity profile obtained during the $\mathrm{x}$ traverse (at the centerline y position) could be replicated to produce velocity profiles at each $y$ location by application of a shape function determined from the $y$-traverse velocity profile data. This function was simply a multiplier based on the ratio of the velocity measured at each $y$ location divided by the velocity at the center location. The full-field velocity data thus obtained are plotted in Fig. 20 for a hydrogen concentration of $3.15 \mathrm{~mol} \%$. The mean velocity across the full field was then determined from the average of all position values. The flow profile in $\mathrm{x}$ showed a double-hump profile, with a lower flow at the center and the two walls. The flow profile in y showed a single peak off the chimney centerline, skewed toward the PAR center.

It is possible to predict the flow velocity through the PAR as a function of the difference in temperature between the PAR outlet and inlet. Integration of the Bernoulli equations in a loop up through the PAR and then down outside the PAR back to the inlet yields the equations of flow, which are essentially a balance between buoyancy forces and flow losses in the PAR. The ambient fluid outside the PAR has a constant temperature that is approximately equal to the PAR inlet temperature. The temperature inside the PAR increases approximately linearly as it rises through the catalyst plates; is relatively constant as it rises in the chimney; and then the gas 
discharges as a buoyant jet with a temperature greater than the ambient temperature outside the PAR. The net buoyant force is

$$
\Delta P=\Delta \rho g\left(H_{c}+\frac{1}{2} H_{p}\right)=\Delta \rho g \quad H_{e f f}
$$

where

$\Delta P=$ differential pressure between chimney inlet and outlet

$\Delta \rho=$ density difference between the fluid outside the chimney and the buoyant jet

$g=$ gravitational constant $\left(9.81 \mathrm{~m} / \mathrm{s}^{2}\right)$

$H_{\mathrm{c}}=$ height of the chimney above the plates $(0.8 \mathrm{~m})$

$H_{\mathrm{p}}=$ height of the plates $(0.2 \mathrm{~m})$

$H_{\text {eff }}=$ effective height of the PAR $(0.9 \mathrm{~m})$

Since the effect of the composition change from the very lean hydrogen combustion on the density is relatively small, the density difference occurs mostly as a result of the temperature change, therefore,

$$
\Delta \rho \cong \rho \frac{\Delta T}{T}
$$

where

$\rho=$ average mixture density

$\Delta T=$ temperature difference between PAR inlet and outlet

$T=\mathrm{PAR}$ average absolute gas temperature

The total volumetric flow, $Q$, is related to the pressure drop by

$$
\Delta P=\frac{1}{2} k \rho V^{2}=\frac{1}{2} k \rho\left(\frac{Q}{A}\right)^{2}
$$

where

$A=$ PAR exit flow area $\left(0.059 \mathrm{~m}^{2}\right)$

$k=$ total loss coefficient (4.4)

The flow losses occur as a result of the entrance contraction at the catalyst plate slots $\left(k_{\text {inlet }}=\right.$ $0.5)$, friction losses for flow along the slots $\left(k_{\text {slot }}=2.0\right)$, exit losses from the slots due to the sudden expansion of area $\left(k_{\text {slot exit }}=0.6\right)$, contraction losses due to the chimney transition $\left(k_{\text {transition }}=0.3\right)$, and the losses due to the sudden expansion of area the chimney exit $\left(k_{\text {exit }}=\right.$ 1.0). The loss coefficients were estimated using fluid dynamics textbooks and the Crane pipe design handbook. The total loss coefficient, $k$, is estimated to be 4.4 .

Rearranging Eqs. 5, 6, and 7 yields the velocity prediction.

$$
V=\left(2 g \frac{h_{e f f}}{k} \frac{\Delta T}{T}\right)^{\frac{1}{2}}
$$


The velocity prediction based on Eq. 8 and the PP-1 PAR $\Delta T$ is plotted in Fig. 19. The estimate of 4.4 for the total loss coefficient is probably close, since the velocity prediction is in good agreement with the data. Figure 19 shows the gas velocity decreasing as a function of time due to the PAR slowing down as the hydrogen concentration decreases.

\section{V.K. PAR Energy Balance}

Once steady state has been achieved, the energy release from the hydrogen combustion should balance the sensible heating of the volumetric flow through the PAR. This provides an independent verification of the PAR flow based on an energy balance. An estimate of the PAR heating rate using the energy release from the hydrogen combustion is simply the measured hydrogen depletion rate times the enthalpy of the reaction of hydrogen:

$$
q_{D P R}=D P R \Delta H_{\text {hydrogen }} 3600 \mathrm{~s} / \mathrm{hr}
$$

where

$q_{\mathrm{DPR}}=$ heating rate from hydrogen combustion (w)

$D P R=$ hydrogen depletion rate $((\mathrm{kg} / \mathrm{hr})$

$\Delta H_{\text {hydrogen }}=$ lower enthalpy of the reaction of hydrogen $(1.19 \mathrm{e} 08 \mathrm{~J} / \mathrm{kg})$

The PAR heat generation rate, based on the measured flow rate and temperature drop:

where

$$
q_{\text {sensible }}=\dot{m}_{C_{p}} \Delta T=\rho A V C_{p} \Delta T
$$

$\dot{m}=$ mass flow rate of gas through the PAR $\left(\mathrm{m}^{3} / \mathrm{s}\right)$

$\rho=$ average density of the gas mixture through the PAR $\left(-2.5 \mathrm{~kg} / \mathrm{m}^{3}\right)$

$A=$ flow area $\left(0.059 \mathrm{~m}^{3}\right)$

$V=$ PAR gas flow velocity $(\mathrm{m} / \mathrm{s})$

$c_{\mathrm{p}}=$ average specific heat of the gas mixture $(-1.02 \mathrm{~kJ} / \mathrm{kg} / \mathrm{K})$

$\Delta T=$ temperature difference of the gas between PAR inlet and exit

Equation 10 was implemented using velocities calculated by Eq.8, and gas mixture average properties were determined from time-dependent gas temperature and concentration measurements at the PAR inlet and exit. Equation 9 used the hydrogen depletion rate measured during the steady-state operation from $5.4 \mathrm{hr}$ to the end of the test. PAR heating rates (as a function of hydrogen concentration) from both methods are compared in Fig. 21 . Good agreement was obtained, providing confidence in the velocity prediction based on the PAR $\Delta T$.

Figure 22 compares velocity calculated using Eq. 8 and the measured PAR PP-1 $\Delta T$ data with the velocity measured by the pitot tubes (PT) during the four traverses. The PT data point at about $0.8 \mathrm{~mol} \%$ hydrogen should be considered somewhat unreliable, since the second PT had already dropped out due to the low flow. A velocity prediction is also plotted, based on Eq. 8 and assuming the temperature correlation $\left(-46 \mathrm{~K}\right.$ PAR $\left.\Delta T / 1 \% \mathrm{H}_{2}\right)$ determined from previous experiments. The velocity correlation should be scale independent, i.e., the velocity for a fullscale PAR should be identical to the 1/8 scale PAR (assuming negligible scaled heat losses and a configuration identical to the PAR design tested at SNL). 
The 1/8 scale PAR volumetric flow rate (velocity data times the flow area, $0.059 \mathrm{~m}^{2}$ ) is also plotted in Fig. 22. The full-scale PAR volumetric flow rate should be approximately eight times that determined in the PP-1 experiment, based on the Sher model assumption that the flow rate correlation is scale dependent. Figure 22 also shows that the volumetric flow rate predicted by Eq. 2 is less than the calculated flow rate based on the PP-1 velocity measurements. The difference is probably due to the additional chimney section which was not used in the EPRI tests).

\section{CONCLUSIONS}

Performance tests of a scaled passive autocatalytic recombiner were performed in the Surtsey test vessel at Sandia National Laboratories. The following conclusions can be drawn: The PAR started immediately with low concentrations of hydrogen $(0.2 \mathrm{~mol} \%)$ in cold air atmospheres. The PAR startup was delayed about $2 \mathrm{hr}$ in a test with a low concentration of hydrogen $(0.4$ mol\%) in a hot, steamy atmosphere. The PAR started in about $10 \mathrm{~min}$ in hot, steamy atmospheres when exposed to hydrogen concentrations $>1-2 \mathrm{~mol} \%$.

The PAR appeared to generate a convective flow loop in the Surtsey vessel from the PAR outlet to the dome and down the Surtsey wall until it reached a height near the PAR inlet, and then returned to the PAR inlet. The wall array thermocouples located at elevations below the PAR showed little temperature stratification compared with those located above the PAR. The loop appeared to move further downward below the PAR with higher hydrogen concentrations and also with a larger PAR scale. Also, the hydrogen concentrations from the sample point located near the floor showed small decreases over time, compared with the other sample points.

The hydrogen depletion rate is most likely proportional to the PAR scale (defined by the PAR inlet flow area). The Surtsey depletion rate data confirm the depletion rate measurements in earlier work described by the Fischer correlation. The parameter that may seem to affect scale proportionality in the Surtsey tests is the well-mixed assumption in the methodology used to determine the depletion rate. Since it is possible for the PAR to consume hydrogen in a portion of the total vessel volume, the depletion rate calculation can overpredict consumption. The depletion rate measurements then become scale dependent since tests with larger scale and/or higher concentrations would appear to deplete larger pockets of hydrogen within the total vessel volume. Unmixed and well-mixed counterpart experiments show that the depletion rate calculation methodology is valid at 1/8 PAR scale. Mixing effects at $1 / 4$ and $1 / 2$ PAR scale cannot be compared because there are no counterpart data.

Two counterpart experiments were performed to determine if the lack of the hydrophobic coating would cause a startup delay in tests with hot, steamy environments and hydrogen concentrations $>5 \mathrm{~mol} \%$. A startup delay of about 18 min occurred in the test with no coating, compared with the test with the hydrophobic coating, which had a startup delay of $7 \mathrm{~min}$. Counterpart experiments determined that the placement of a PAR near a wall yielded depletion rates smaller than those obtained with the PAR placed in the middle of the facility. The oxygen limit effect was intentionally tested. The PAR depleted hydrogen at very low oxygen concentrations; however, oxygen starvation certainly yielded reduced depletion rates and PAR temperatures (i.e., 
low oxygen concentrations limit the amount of hydrogen that can recombine at rates less than those observed at stoichiometric levels).

Hydrogen was injected at a rate slightly above the PAR consumption to evaluate the hydrogen ignition characteristics of a PAR in a well-mixed air/steam environment with both new and used hydrophobic coating on the catalyst pellets. Four hydrogen deflagration events (burns) occurred in the Surtsey vessel. The burns occurred under closely repeatable conditions of hydrogen concentration and PAR temperature in conjunction with hot particles bursting from the PAR exhaust.

Velocity measurements of the PAR exhaust gas were taken at four different hydrogen concentrations as the PAR recombined and reduced the hydrogen gas in the Surtsey vessel. The data were used to develop a relationship to predict the flow velocity through the PAR as a function of the difference in temperature between the PAR outlet and inlet. The PAR heating rate was then calculated using the velocity prediction. The PAR heating rate was also calculated using the energy release from the measured hydrogen combustion. Good agreement was obtained between the two independent energy methods, providing confidence in the velocity prediction based on the PAR $\Delta T$.

\section{ACKNOWLEDGMENTS}

This work was supported by the U.S. Nuclear Regulatory Commission and was performed at Sandia National Laboratories. Sandia is a multiprogram laboratory operated by Sandia Corporation, a Lockheed Martin Company, for the U.S. Department of Energy under Contract DE-ACO4-94AL85000.

\section{REFERENCES}

1. Electric Power Research Institute (EPRI), 1997a. Effects of Inhibitors and Poisons on the Performance of Passive Autocatalytic Recombiners (PARs) for Combustion Gas Control in ALWRs, Electric Power Research Institute, Advanced Light Water Reactor Program Report, Palo Alto, CA, May 22, 1997.

2. O. BRAILLARD, S. GUIEU, J. HOSLER, and G. SLITER, 1997. Tests of Passive Autocatalytic Recombiners (PARs) for Combustion Gas Control in Nuclear Power Plants, paper presented at ANS $2^{\text {nd }}$ International Topical Meeting on Advanced Reactor Safety.

3. Electric Power Research Institute (EPRI), 1993. Qualification of Passive Autocatalytic Recombiners for Combustion Gas Control in ALWR Containments," Electric Power Research Institute, Advanced Light Water Reactor Program Report, Palo Alto, CA.

4. NIS Control Module for Hydrogen Removal in Containment Atmosphere, Information Brochure, 1992. NIS Ingenieurgesellschaft Mbh, Hanau, Germany.

5. T. K. BLANCHAT, M. D. ALLEN, M. PILCH, and R. T. NICHOLS, 1994. Experiments to Investigate Direct Containment Heating Phenomena with Scaled Models of the Surry Nuclear 
Power Plant, NUREG/CR-6152, SAND93-2519, Sandia National Laboratories, Albuquerque, NM.

6. T. K. BLANCHAT and A. MALLIAKOS, 1998. Performance Testing Of Passive Autocatalytic Recombiners, NUREG/CR-6580, SAND97-2632, Sandia National Laboratories, Albuquerque, NM.

7. T. K. BLANCHAT, 1998. Report on PAR Flow Measurements: The PP-1 Test, Letter report to A. Malliakos (USNRC), Sandia National Laboratories, Albuquerque, NM.

8. T. K. BLANCHAT, 1998. Report on Ignition Behavior of PARs: The PP-2 Test, Letter report to A. Malliakos (USNRC), Sandia National Laboratories, Albuquerque, NM.

9. K. FISCHER, 1995. Qualification of a Passive Catalytic for Hydrogen Mitigation," Nuclear Technology, Vol. 112.

10. R. SHER. J. LI, and D.E. LEAVER, 1995, Models for Evaluating the Performance of Passive Autocatalytic Recombiners (PARs), paper presented at 1995 National Heat Transfer Conference, ANS Proceedings HTC, Vol. 8. 
Table 1

PAR Test Matrix

\begin{tabular}{|c|c|c|c|}
\hline Test & Purpose & Scale & Atmosphere \\
\hline 1 & $\begin{array}{l}\text { Startup in Air } \\
\text { Find } \mathrm{X}_{\mathrm{H} 2 \text {, air start }}\end{array}$ & $1 / 2$ & $\begin{array}{l}2 \text { bar air, no steam, } \\
0.2 \%, 0.4 \%, 0.6 \% \mathrm{H}_{2} \ldots \mathrm{X}_{\mathrm{H} 2 \text {, air start }}\end{array}$ \\
\hline 2 & Performance at Startup & $1 / 2$ & 2 bar air, no steam, inject $X_{\mathrm{H} 2 \text {, start }}$ (single injection) \\
\hline 3 & $\begin{array}{l}\text { Startup in Air/Steam } \\
\text { Find } \mathrm{X}_{\mathrm{H} 2 \text {, air/steam start }}\end{array}$ & $1 / 2$ & $\begin{array}{l}1 \text { bar air, } 1 \text { bar steam, } \\
0.2 \%, 0.4 \%, 0.6 \% \mathrm{H}_{2} \ldots \mathrm{X}_{\mathrm{H} 2 \text {, airsteam start }}\end{array}$ \\
\hline 4 & $\begin{array}{l}\text { Scale/depletion rate } \\
\text { without startup transient }\end{array}$ & $1 / 2$ & $\begin{array}{l}1 \text { bar air, } 1 \text { bar steam, continuous injection to maintain } \\
1.4 \% \mathrm{H}_{2} \text { until PAR reaches steady state (SS) }\end{array}$ \\
\hline 5 & $\begin{array}{l}\text { Scale/depletion rate } \\
\text { without startup transient }\end{array}$ & $1 / 4$ & $\begin{array}{l}1 \text { bar air, } 1 \text { bar steam, continuous injection to maintain } \\
1.4 \% \mathrm{H}_{2} \text { until PAR reaches SS (same as PAR-4) }\end{array}$ \\
\hline 6 & $\begin{array}{l}\text { Scale/depletion rate without startup } \\
\text { transient }\end{array}$ & $1 / 8$ & $\begin{array}{l}1 \text { bar air, } 1 \text { bar steam, continuous injection to maintain } \\
1.4 \% \mathrm{H}_{2} \text { until PAR reaches SS (same as PAR-4) }\end{array}$ \\
\hline 7 & $\begin{array}{l}\text { Performance, } \\
\text { (this test will destroy the hydrophobic } \\
\text { coating) }\end{array}$ & $1 / 8$ & $\begin{array}{l}1 \text { bar air, } 1 \text { bar steam, continuous injection to maintain } \\
7.0 \% \mathrm{H}_{2} \text { until PAR reaches SS }\end{array}$ \\
\hline $\begin{array}{l}8 \\
8 \mathrm{R}\end{array}$ & $\begin{array}{l}\text { Performance } \\
\text { (without the hydrophobic coating) }\end{array}$ & $1 / 8$ & $\begin{array}{l}1 \text { bar air, } 1 \text { bar steam, continuous injection to maintain } \\
7.0 \% \mathrm{H}_{2} \text { until PAR reaches SS (same as PAR-7) }\end{array}$ \\
\hline 9 & $\begin{array}{l}\text { PAR at wall to determine impact on } \\
\text { PAR performance (use new catalyst } \\
\text { with intact hydrophobic coating) }\end{array}$ & $1 / 8$ & $\begin{array}{l}1 \text { bar air, } 1 \text { bar steam, continuous injection to maintain } \\
1.4 \% \mathrm{H}_{2} \text { until PAR reaches SS (same as PAR-6) }\end{array}$ \\
\hline 10 & $\begin{array}{l}\text { Hydrogen ignition by PAR } \\
\text { PAR back to vessel centerline }\end{array}$ & $1 / 8$ & 1 bar air, 1 bar steam, quick injection of $10 \% \mathrm{H}_{2}$ \\
\hline 12 & $\begin{array}{l}\text { Performance/scale/depletion } \\
\text { without startup transient }\end{array}$ & $1 / 4$ & $\begin{array}{l}1 \text { bar air, } 1 \text { bar steam, continuous injection to maintain } \\
7.0 \% \mathrm{H}_{2} \text { until PAR reaches SS (same as PAR-7) }\end{array}$ \\
\hline $\begin{array}{l}13 \\
13 R \\
\end{array}$ & $\begin{array}{l}\text { Performance/scale/depletion } \\
\text { without startup transient }\end{array}$ & $1 / 2$ & $\begin{array}{l}1 \text { bar air, } 1 \text { bar steam, continuous injection to maintain } \\
7.0 \% \mathrm{H}_{2} \text { until PAR reaches SS (same as PAR-7) }\end{array}$ \\
\hline $\begin{array}{l}\text { demo1 } \\
\text { demo2 } \\
\text { demo3 }\end{array}$ & $\begin{array}{l}\text { Performance/depletion rate in a well- } \\
\text { mixed environment }\end{array}$ & $1 / 8$ & 2 bar air, no steam, continuous mixing, $6.0-10.0 \% \mathrm{H}_{2}$ \\
\hline 14 & $\begin{array}{l}\text { Performance/depletion rate in a well- } \\
\text { mixed environment } \\
\text { (new catalyst) }\end{array}$ & $1 / 8$ & 2 bar air, no steam, continuous mixing, $2.0 \% \mathrm{H}_{2}$ \\
\hline 15 & $\begin{array}{l}\text { Performance/depletion rate in a well- } \\
\text { mixed environment } \\
\text { (same catalyst as PAR-14) }\end{array}$ & $1 / 8$ & 2 bar air, no steam, continuous mixing, $2.0 \% \mathrm{H}_{2}$ \\
\hline 16 & $\begin{array}{l}\text { Performance in low oxygen } \\
\text { environment } \\
\text { (same catalyst as PAR-14) }\end{array}$ & $1 / 8$ & $\begin{array}{l}2 \text { bar nitrogen, no steam, continuous mixing, } \\
2.0-4.0 \% \mathrm{H}_{2}, 0.0-4.0 \% \mathrm{O}_{2}\end{array}$ \\
\hline $\mathrm{PP}-1$ & PAR flow measurements & $1 / 8$ & 2 bar air, no steam, continuous mixing, $1.0-3.0 \% \mathrm{H}_{2}$ \\
\hline PP-2 & $\begin{array}{l}\text { Hydrogen ignition by PAR, new and } \\
\text { used hydrophobic coating }\end{array}$ & $1 / 8$ & $\begin{array}{l}1 \text { bar air, } 1 \text { bar steam, slow continuous injection and } \\
\text { mixing to achieve SS until PAR ignites mixture }\end{array}$ \\
\hline
\end{tabular}


Table 2

Initial Conditions Prior to the Hydrogen Ignition Events

\begin{tabular}{|l|l|l|l|l|l|l|l|l|l|l|}
\hline PAR & $\begin{array}{l}\mathrm{H}_{2} \\
(\%)\end{array}$ & $\begin{array}{l}\mathrm{O}_{2} \\
(\%)\end{array}$ & $\begin{array}{l}\text { Steam } \\
(\%)\end{array}$ & $\begin{array}{l}P_{\text {vessel }} \\
(\mathrm{MPa})\end{array}$ & $\begin{array}{l}T_{\text {vessel }} \\
(\mathrm{K})\end{array}$ & $\begin{array}{l}T_{\text {catalyst }} \\
(\mathrm{K})\end{array}$ & $\begin{array}{l}\Delta T \\
(\mathrm{~K})\end{array}$ & Scale & $\begin{array}{l}\Delta P \\
(\mathrm{MPa})\end{array}$ & Notes \\
\hline 8 & 11.0 & 9 & 48 & 0.26 & 392 & 1140 & 280 & $1 / 8$ & 0.30 & 1 \\
\hline 10 & 13.0 & 13 & 48 & 0.25 & 380 & 950 & 70 & $1 / 8$ & 0.39 & \\
\hline 13 & 4.4 & 12 & 50 & 0.24 & 378 & 880 & 20 & $1 / 2$ & 0.02 & $1,2,3$ \\
\hline 13 & 5.5 & 12 & 50 & 0.26 & 380 & 780 & 10 & $1 / 2$ & 0.06 & $1,2,3,5$ \\
\hline 13 & 4.0 & 11.5 & 55 & 0.30 & 415 & 800 & 160 & $1 / 2$ & 0.04 & $1,2,3$ \\
\hline $13 \mathrm{R}$ & 6.0 & 15 & 46 & 0.25 & 380 & 740 & 90 & $1 / 2$ & 0.05 & $1,2,3$ \\
\hline $13 \mathrm{R}$ & 6.0 & 14 & 46 & 0.27 & 410 & 880 & 140 & $1 / 2$ & 0.03 & $1,2,3$ \\
\hline demo1 & 7.0 & 20 & 6 & 0.24 & 320 & 840 & 170 & $1 / 8$ & 0.15 & 2,6 \\
\hline demo1 & 6.8 & 15 & 1 & 0.24 & 330 & 820 & 160 & $1 / 8$ & 0.07 & $2,6,7$ \\
\hline demo1 & 8.5 & 13 & 2 & 0.25 & 340 & 1020 & 220 & $1 / 8$ & 0.30 & $2,5,7,8$ \\
\hline demo2 & 11.0 & 19 & 0 & 0.22 & 310 & 680 & 35 & $1 / 8$ & 0.47 & $2,4,7,8$ \\
\hline demo3 & 6.0 & 19 & 1 & 0.23 & 320 & 850 & 160 & $1 / 8$ & 0.04 & $6,7,8$ \\
\hline PP-2, 1 & 6.1 & 13.5 & 48.0 & 0.25 & 390 & 942 & 290 & $1 / 8$ & 0.14 & $2.11 \mathrm{hr}$ \\
\hline PP-2,2 & 6.3 & 12.5 & 52.5 & 0.29 & 398 & 948 & 308 & $1 / 8$ & 0.18 & $3.00 \mathrm{hr}$ \\
\hline PP-2,3 & 6.8 & 12.2 & 55.0 & 0.26 & 399 & 968 & 323 & $1 / 8$ & 0.17 & $4.65 \mathrm{hr}$ \\
\hline PP-2,4 & 5.9 & 11.2 & 58.5 & 0.28 & 404 & 893 & 273 & $1 / 8$ & 0.10 & $5.30 \mathrm{hr}$ \\
\hline $\begin{array}{l}\text { Average } \\
\pm\end{array}$ & 6.3 & & & & & 938 & 299 & & & \\
\hline $\begin{array}{l}\text { Std. Dev. } \\
\text { in PP-2 }\end{array}$ & 0.4 & & & & & \pm & \pm & & & \\
\hline
\end{tabular}

Notes: (1) during $\mathrm{O}_{2}$ add, (2) during $\mathrm{H}_{2}$ add, (3) white particles floating in vessel, (4) flame front from floor, (5) flame front from PAR outlet, (6) flame front from ceiling, (7) no hot-wire anemometer, (8) no quartz lamps. 


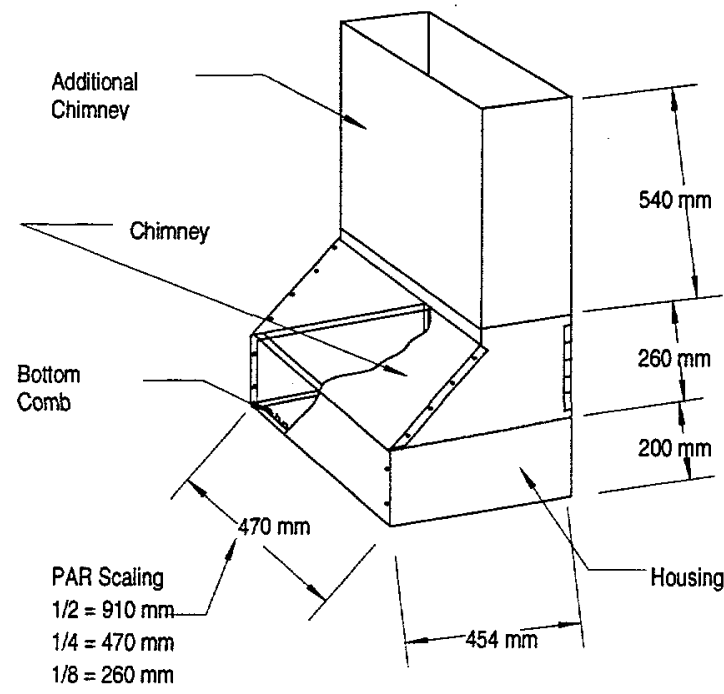

Fig. 1. Multiscale PAR test module housing.

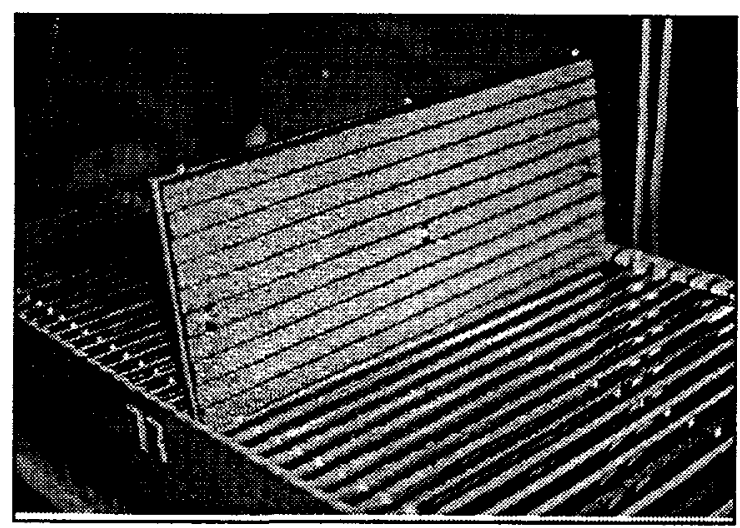

Fig. 2. Cartridges held in a vertical configuration (chimney removed).

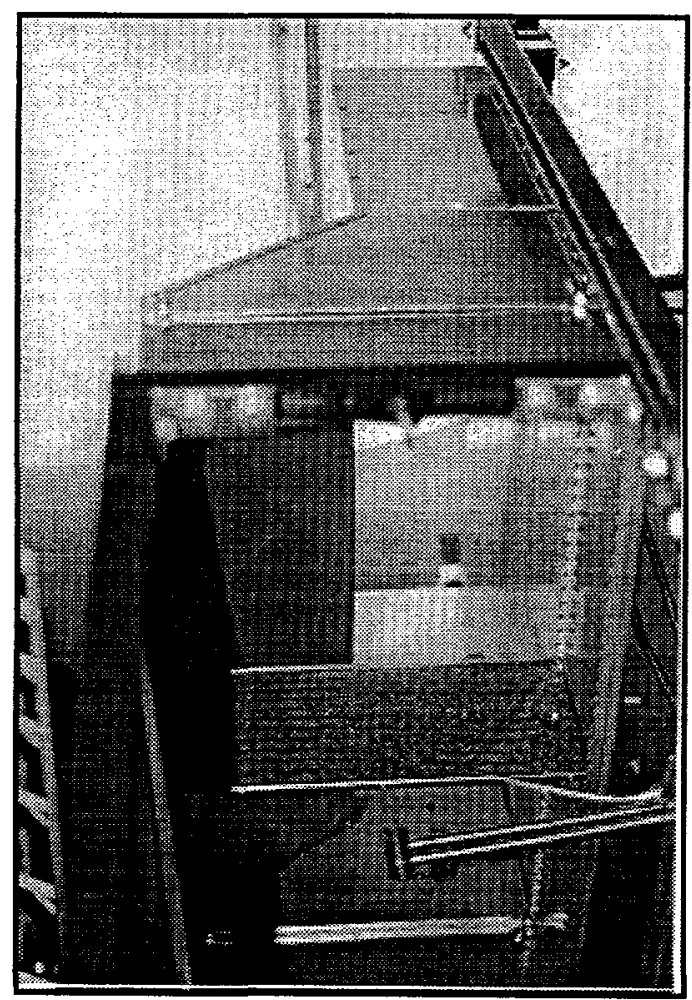

Fig. 3. Bottom view through PAR housing.

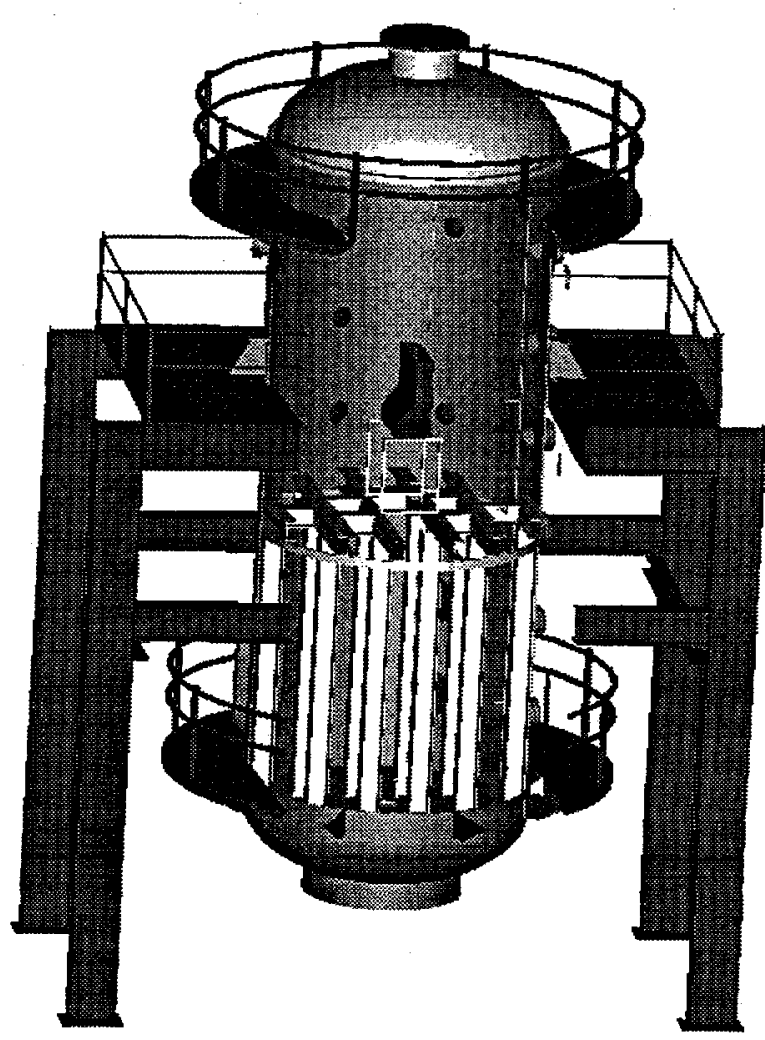

Fig. 4. PAR location in the Surtsey vessel.

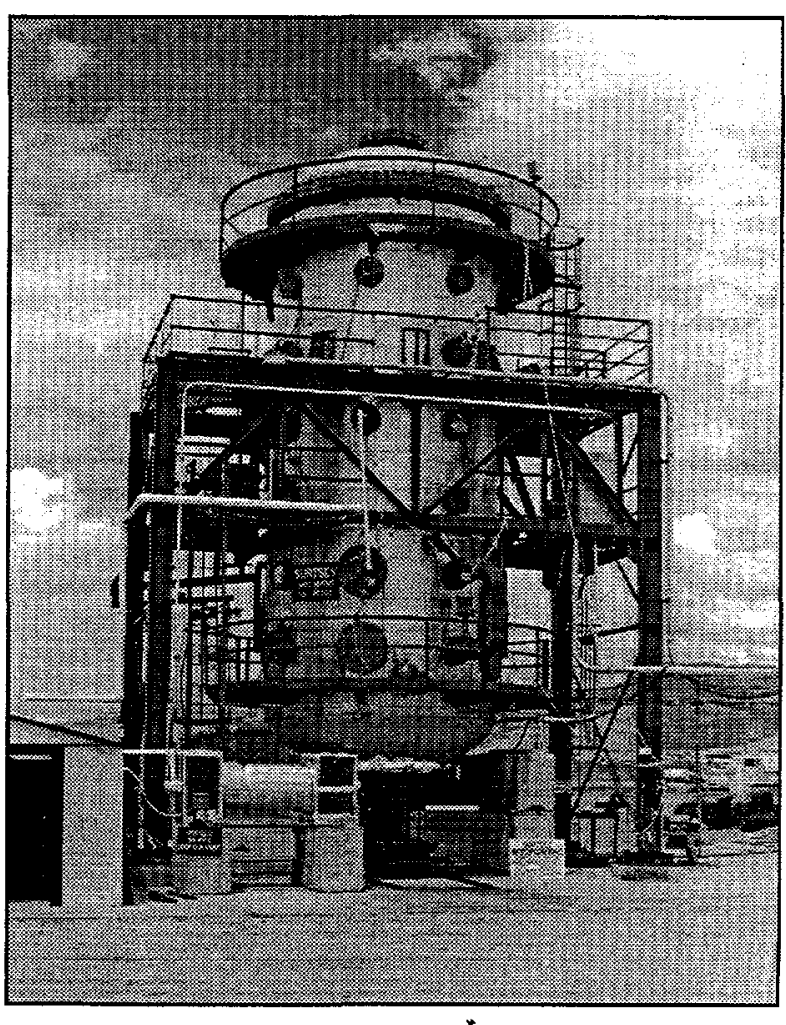

Fig. 5. The Surtsey vessel. 


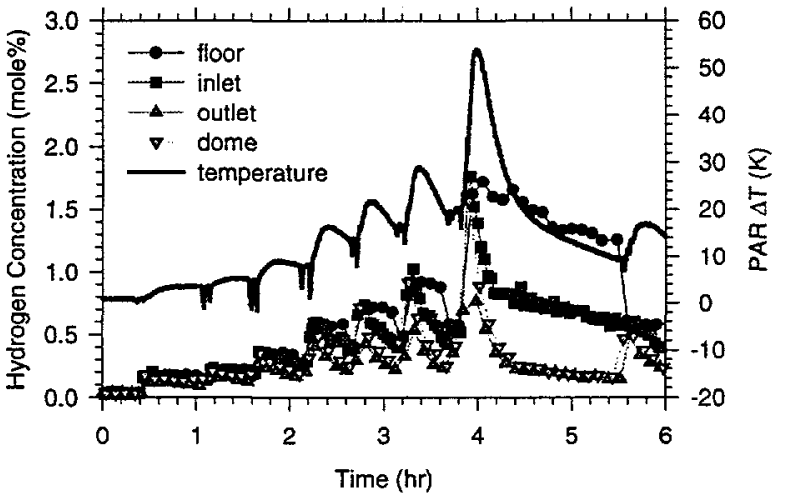

Fig. 6. Hydrogen concentration compared with $\Delta T$ in PAR-2.

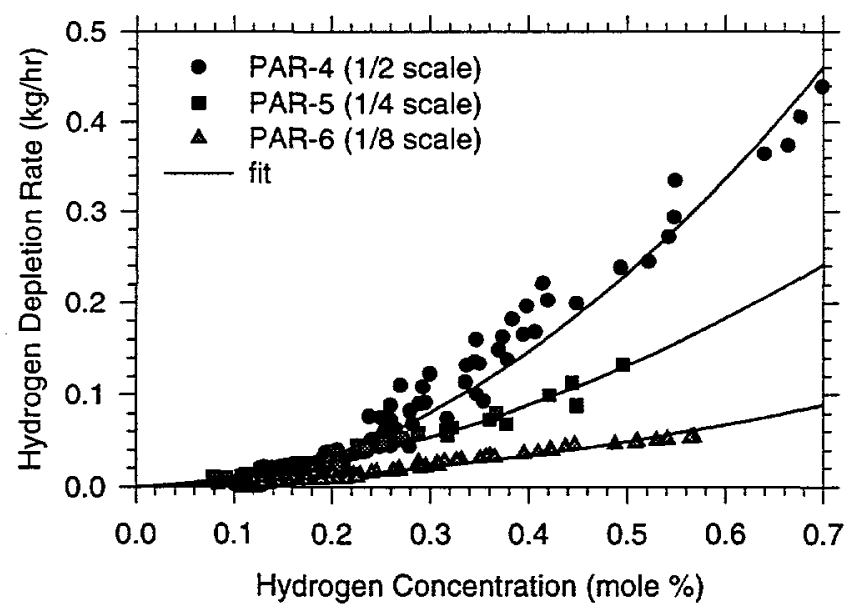

Fig. 7. PAR performance at low hydrogen concentrations.



Fig. 8. PAR performance at high hydrogen concentrations.

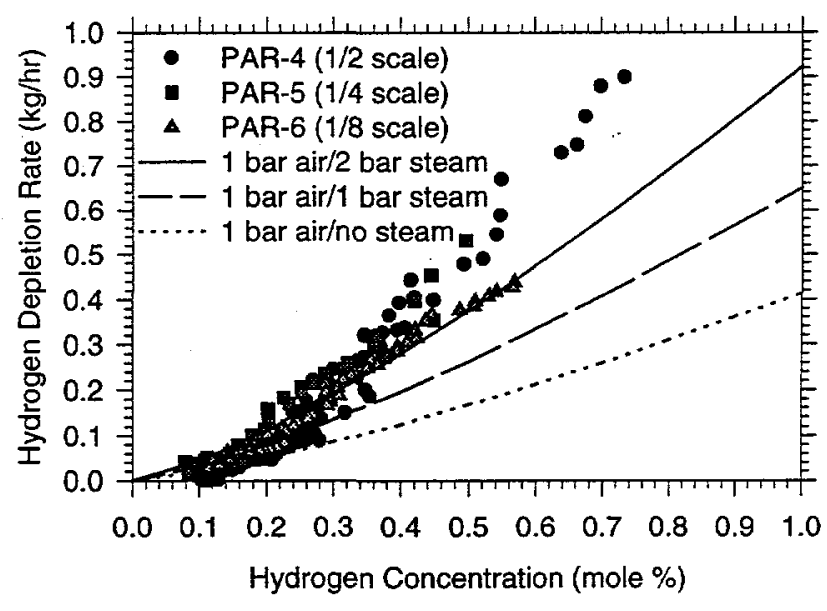

Fig. 9. Normalized depletion rate at low hydrogen concentrations. 


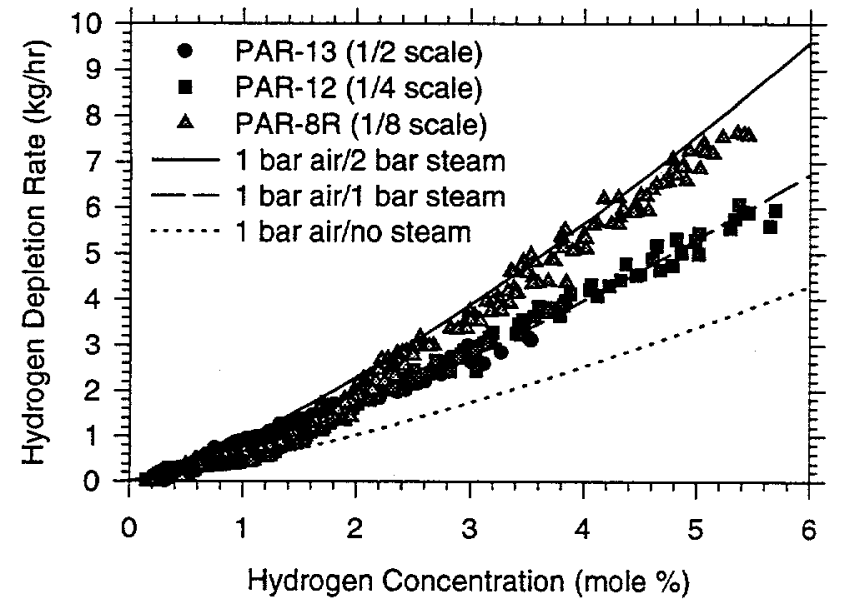

Fig. 10. Normalized depletion rate at high hydrogen concentrations.

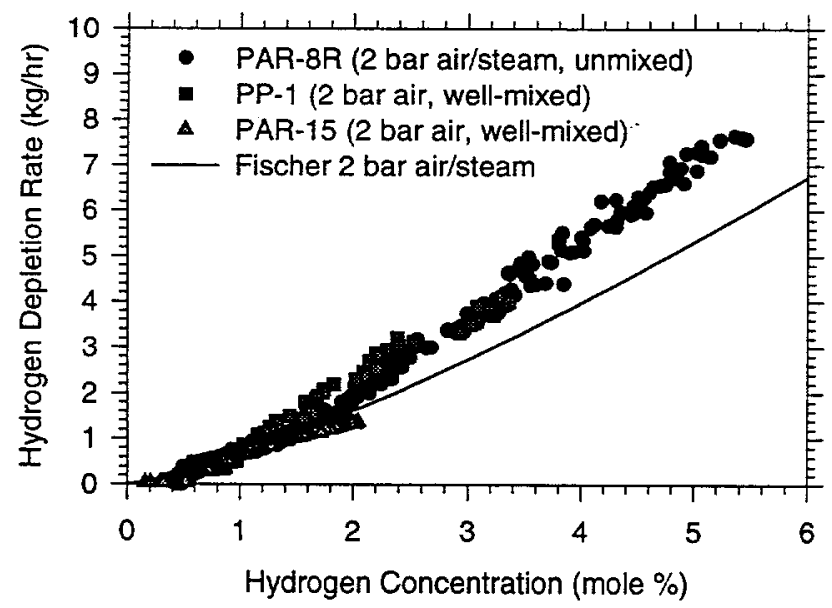

Fig. 11. Unmixed vs. well-mixed depletion rates.

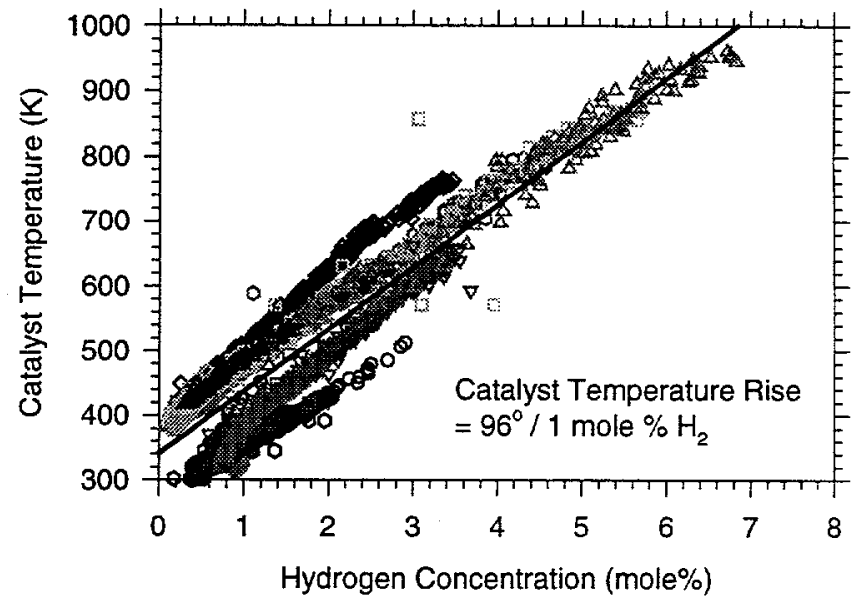

Fig. 12. PAR catalyst temperature as a function of hydrogen concentration.

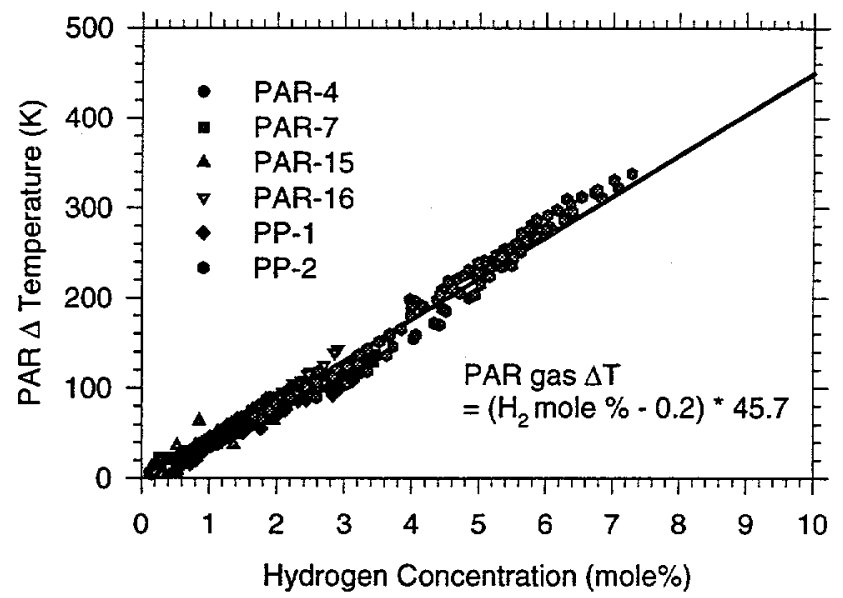

Fig. 13. PAR gas $\Delta T$ as a function of hydrogen concentration. 


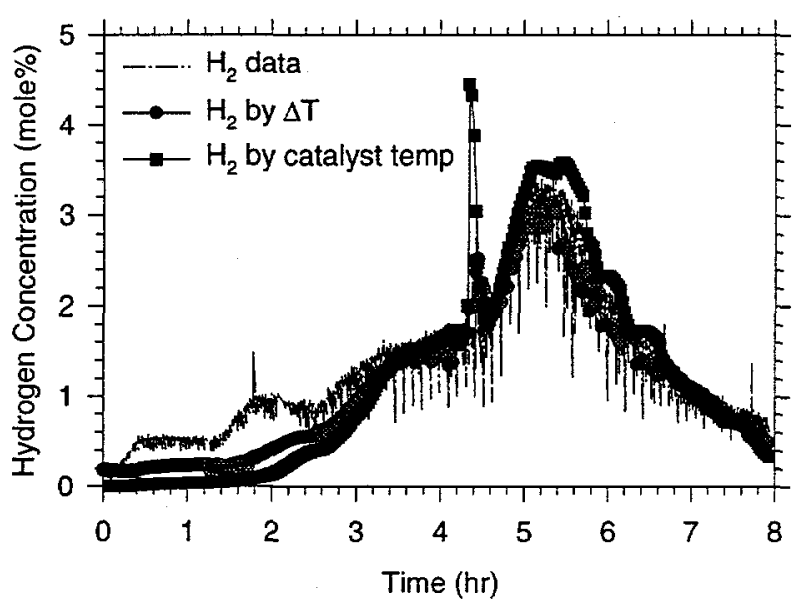

Fig. 14. PP-1 hydrogen measurement and prediction.

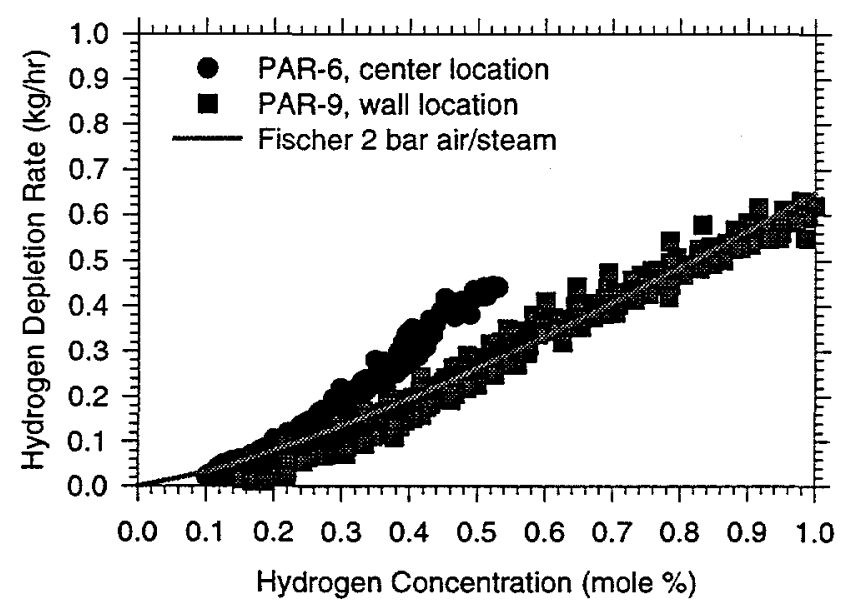

Fig. 15. Hydrogen depletion rate and the wall effect.

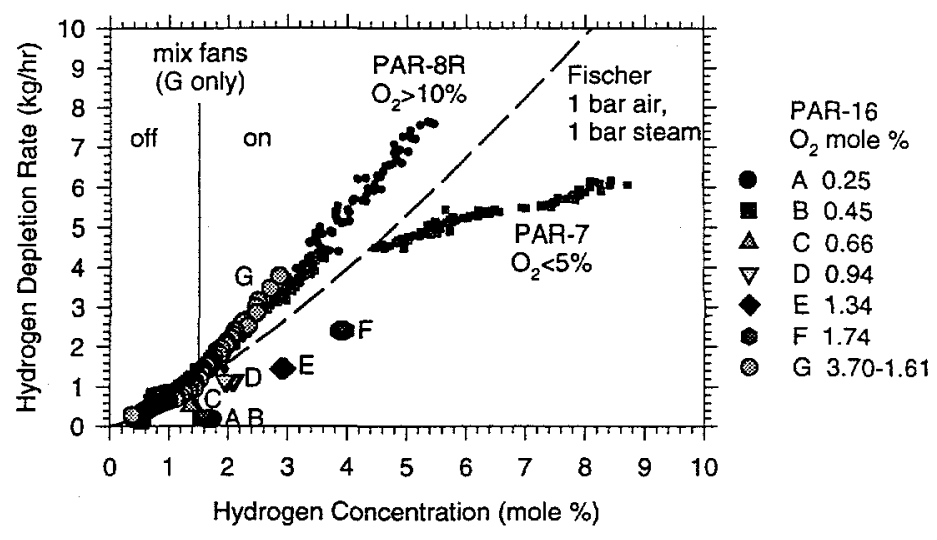

Fig. 16. Hydrogen depletion rate and the $\mathrm{O}_{2}$ limit.

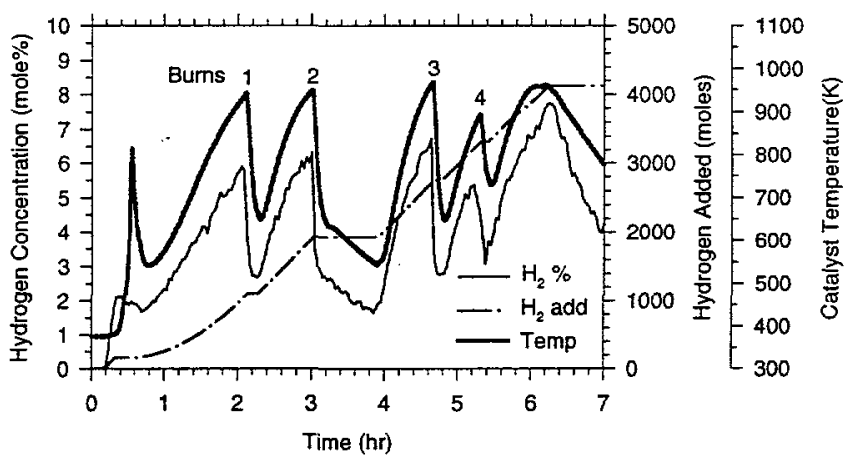

Fig. 17. Hydrogen history and catalyst temperature in the PP-2 ignition test. 


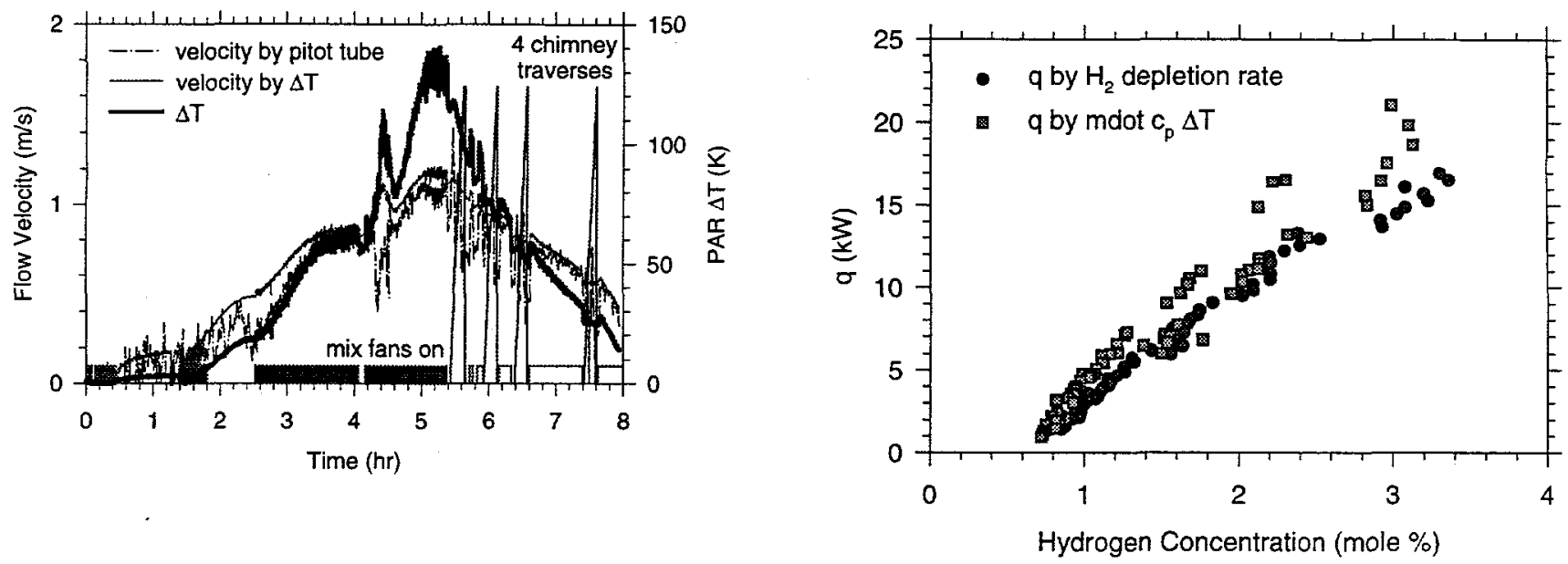

Fig. 19. Velocity and temperature vs. time in PP-1.
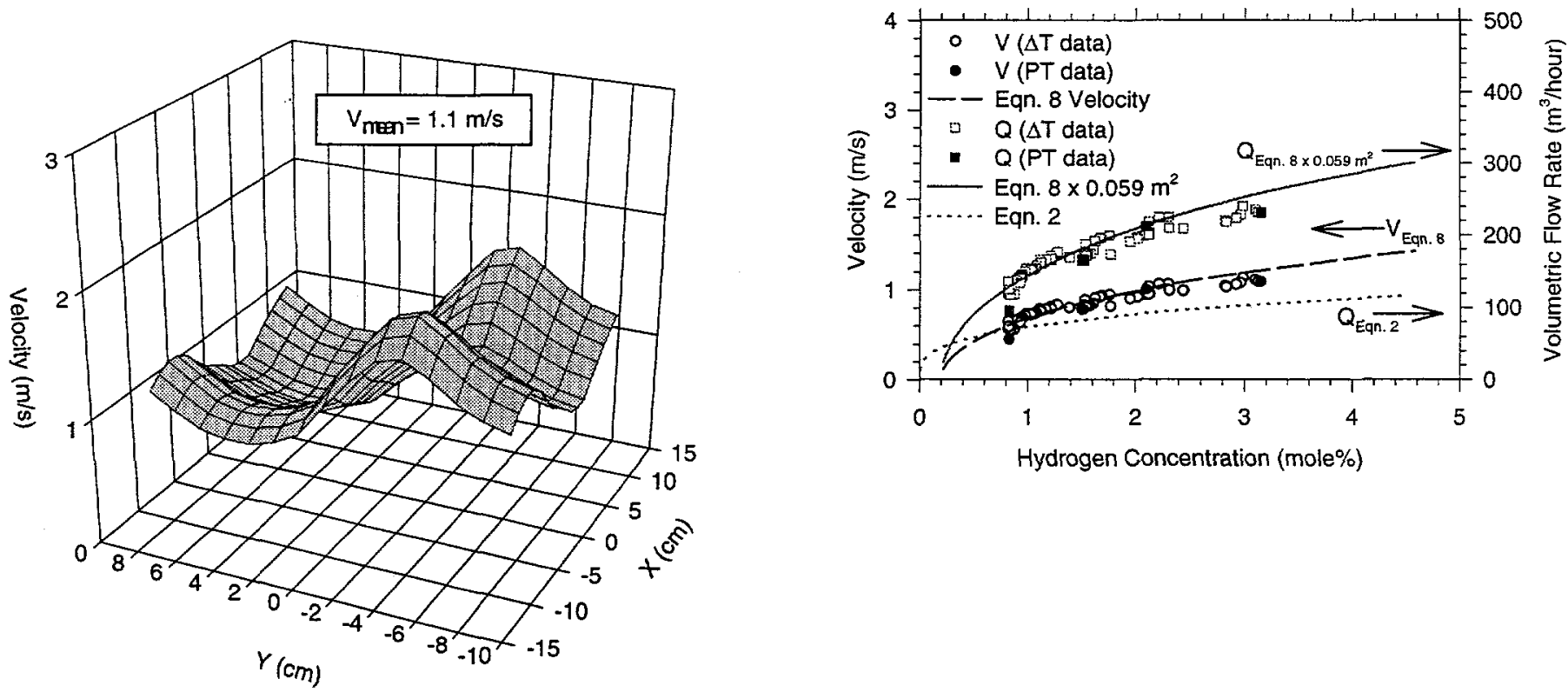

Fig. 20. Full-field velocity at the chimney exit in PP-1 at $\sim 3$ mol\% hydrogen.

Fig. 21. PAR heating by two methods.

Fig. 22. PAR gas velocity and volumetric flow rate as a function of hydrogen concentration. 